\title{
Everyday-ing Health Literacy and the Imperative of Health Communication: A Critical Agenda
}

\author{
Eric Po keung Tsang and Dennis Lai Hang Hui
}

\begin{abstract}
Health literacy is an increasingly important issue amongst scholars of health studies and medical practitioners. This essay seeks to understand how health knowledge is co-constructed by different agencies and the role of health communication in this process can contribute to everyday-ing health literacy. In addition, this essay attempts to understand how health communication becomes an everyday practice in identifying disease-specific needs.
\end{abstract}

Keywords Health literacy • Policy-making • Everyday health needs

\section{Background}

To-date, discourses related to health and illness are no longer confined within hospitals and other medical establishments; they have become an everyday, interactive discourse which helps define our way to perceive health issues. This chapter provides an everyday perspective towards the idea of human communication and locates the importance of health literacy-the production and consumption of health-related knowledge and information (Kickbusch and Maag 2008) -in enhancing the quality of public health service. Firstly, we examine the importance of health literacy in establishing the basis of health communication. As recognised by the World Health Organization (n.d.), health literacy shall be playing a critical role in encouraging multi-stakeholder dialogue in empowering individuals and communities. How to put health literacy into policy-based, knowledge-informed action is an important issue that health administrators should consider seriously. Second, we try to look at how health communication is facilitated by the changing

E.P.k. Tsang ( $ه)$ - D.L.H. Hui

The Education University of Hong Kong, Lo Ping Road, Tai Po, Hong Kong

e-mail: etsang@eduhk.hk

(C) The Author(s) 2016

M.E. Robertson (ed.), Communicating, Networking: Interacting,

SpringerBriefs in Global Understanding, DOI 10.1007/978-3-319-45471-9_7 
modes of communication and compare different media-technological options in knowledge construction across different sectors. For the purpose of this chapter, we look specifically at how the increasing concern for health risk offers possibilities for a networked way of knowledge construction with reference to three aspects and the case of mental health literacy. Overall, we attempt to shed light on how information and communications technology can facilitate the promotion of health literacy.

\section{A Conceptual Background About Health Literacy and Health Communication}

We begin with the idea of health literacy. Essentially, health literacy refers to the competence to make informed decisions on a wide range of health-related matters such as nutrition, medication, choice of medical services, and ways to minimise exposure to illnesses (Kickbusch and Maag 2008). With the development of information technology and the increasing awareness of the imperatives of public health education, health literacy has become an emerging governance agenda. A new policy discourse has emerged which is driven by participation of different stakeholders such as medical professionals, community networks, research institutions, and individuals. At the normative level, health literacy is about how to create a knowledge discourse which can ensure convergence of values, understandings and expectations about health-related matters. In this connection, World Health Organization points to the need to cultivate 'the cognitive and social skills' in broadening the basis of participation (WHO n.d.). In operationalising the concept, Nutbeam (2000) points to the need to pay attention to functional, interactive and critical aspects of health literacy. Whereas the functional aspect refers to individual's ability to understand basic health information, the interactive aspect refers to the increasing autonomy of individuals to reflect on different health information (ibid). Critical health literacy, meanwhile, transcends those functional parameters and addresses how individuals and communities are networked in health knowledge construction with their own literacies (ibid).

Meanwhile, what has also been explored in the existing literature are the ways in which health literacy can be promoted. Indeed, the unsatisfactory track-record of promoting health literacy at the global level reflects the failure to integrate health, education and communication together. As emphatically argued by Kickbusch (2001), the imbalance in developing competence in health-related matters reflects the inability to overcome the divide between literacy, communication and knowledge construction. In that connection, there is a need to bring health communication back into the policy discourse.

Health communication, broadly speaking, is about how to create a communicative context for achieving a diversity of health-related purposes. As defined by Ratzan et al. (2004), health communication is "the process through which one person, group, or governmental or private organization uses various communication strategies and channels to educate, motivate, and perpetuate information, skills, and 
behaviours that are generally accepted to benefit (improve) the health of individuals and the public" (p. 398). Originally used in the context of commercial marketing (e.g. the promotion by pharmaceutical companies and insurance companies), health communication has assumed a wider scope of importance (Thomas 2006). Nowadays, health communication becomes an everyday dynamic that defines our understanding about possible options for us to pursue health-related behaviours. For example, health communication is becoming more important in sharing patient information within a jurisdiction (Thomas 2006). Health communication is also widely used in community empowerment (Thomas 2006). In developing economies, for instance, health communications - both formal and informal - have been used to enhance the preparedness towards health crises. In the intellectual context, health communication is shaping the fundamental ways in which health issues are narrated and problematised on a day-to-day basis (Dutta and Zoller 2008). Meanings of health, illness and disease are, accordingly, contingent. A recent edited volume by Hamilton et al. (2014) goes further by contending that health communication is a linguistically situated practice mediated by different contexts and modes of interaction. In this next section, we examine how the advancement of technology has reshaped health communication and the opportunities and challenges associated with promoting health literacy.

\section{Global Diversity in Health Communication and Everyday-ing Health Literacy}

Health communication and health literacy co-evolves with the changing form of media (Hagglund et al. 2009). The role of the traditional media in promoting health literacy has been discussed in the existing body of work. Jorm (2000), for example, discusses the use of traditional media in Defeat Depression Campaign in promoting community awareness towards mental health. How far the momentum for health literacy can be sustained is highly debatable. Scholars thus look to the prospect of the ascendency of e-society as a possibility for offering a new way of health communication. For example, emphasising the importance of interactivity and user-friendliness, the new digital media such as the Internet (especially Web 2.0), social media, digital games, and use of avatars has opened up a wider possibility for a networked way for collaborative construction of health information and knowledge (Prestin and Chou 2014). Laverack (2009) argued that with the increasing availability of low-cost and innovative communication options, the role of the public in health literacy is becoming more proactive. Health literacy is, accordingly, more than helping individuals to make informed decision; it becomes a discursive tool for everyday mobilisation for health development. The Centers for Disease Control and Prevention (CDC) has identified the following aspects of social media 
and the emerging interactive media which allows a more 'credible' form of health communication:

- "Increase the timely dissemination and potential impact of health and safety information.

- Leverage audience networks to facilitate information sharing.

- Expand reach to include broader, more diverse audiences.

- Personalize and reinforce health messages that can be more easily tailored or targeted to particular audiences.

- Facilitate interactive communication, connection and public engagement.

- Empower people to make safer and healthier decisions" (CDC 2011, p. 1).

Based on these aspects, we identify several examples where health communication, health literacy, and the new media coalesce. One of the key observations by public health scholars is that the proliferation of electronic (new) media has led to a 'tectonic shift' in the way people are engaged in processes related to health-related knowledge and information (Hesse et al. 2005). The literature has identified two levels of interaction that the evolving media landscape is impacting on health literacy. On one hand, social media is playing a functional role in expanding the clinical space beyond hospitals and medical establishments. The health care provider is capitalising upon the use of e-technology in forging a closer physicianpatient relationship (Sundar et al. 2011). Communications beyond the face-to-face consultation allow patients to receive and share a wider range of clinical information such as chronic disease self-management (Prestin and Chou 2014) and interventional evaluation (Harrison et al. 2010). Meanwhile, it is reported the use of peer-to-peer (P2P) communication is useful in developing competence in understanding technical information and in accessing relevant health provider. $\mathrm{P} 2 \mathrm{P}$ communication also provides information related to how patients can communicate with medical specialist. The study by Meier et al. (2007) looks, for example, at how cancer patients can develop linguistic competence in everyday communication with the medical expert. These information are, however, 'instructional' and 'pedagogical' (Evans et al. 2009) at best, and may not promote a more dynamic way of promoting health literacy.

Beyond the clinical dimension, the 'digitalisation' of health communication has created opportunities for a dynamic, critical approach for promoting health literacy. We here offer an overview of three possible opportunities. First is the possible dialogue between 'traditional' expert epidemiology and lay epidemiology. Whereas traditional epidemiology refers to how medical professions define the nature of a health problem based on scientific discourse, lay epidemiology refers to how people construct their health risks with reference to their subjectivities and bodily constitutions. Lay epidemiology is thus about how the society becomes a knowledge basis for possible community-based actions for enhancing health. The possible contribution of public knowledge in informing professional action has been documented in different works (Arksey 1994; Davison et al. 1991). Essentially, it provides very important data about the possible cause of health risks (such as 
environmental risks). Whilst some scholars have contested the reliability of public knowledge in ensuring effective public awareness, lay epidemiology becomes a new discourse in exposing conceptions (and misconceptions) about health and disease and provides clues as to what kind of public health intervention is necessary (Laverack 2009). This is made possible by the prevailing use of social media of Twitter, Facebook and other social blogs which has fostered a new socio-political culture of sharing.

Another key opportunity is the use of social media in enhancing community preparedness for health disasters. In the first place, the use of geographic information system (GIS) becomes more prevalent in constructing necessary knowledge for emergency medicine. The Ready New York initiative was developed by the New York City Government in allowing residences to develop their own plans of evacuation in cases of natural or human-made disasters (Zarcadoolas and Pleasant 2009). Cromley and McLafferty (2012) point to the geospatial mode of health communication which can allow health authorities, community leaders and residents to identify the possible 'hotspots' for critical intervention. For example, the US Department of Homeland Security's Science \& Technology Directorate, capitalising upon the proliferation of smartphones and other GPS technologies, has introduced the Social Media Alert and Response to Threats to Citizens (SMART-C) programme. The programme, accordingly, "aims to develop citizens' participatory sensing capabilities for decision support throughout the disaster life cycle via a multitude of devices (such as smartphones) and modalities (MMS messages, Web portals, blogs, tweets, and so on)" (Adam et al. 2012, p. 92). At the same time, the use of online virtual gaming is popularising emergency health knowledge. The Centers for Disease Control and Prevention has recently made use of Zombie Preparedness to create a virtual community for engaging public in developing emergency preparedness (CDC 2015).

The third opportunity is the usage of new media in different health campaigns can equip community members for enhancing linguistic and knowledge capacities. Although the traditional printed media has a relatively long history in contributing to the success of many health campaigns, the new media provides a more dynamic and robust way of engaging a wider scope of audience. Yan Tian's study on the impact of Web 2.0 arrives at the conclusion that the media has a positive impact on the audience's understanding about issues related to organ donation (Tian 2010). Donors' families, recipients and activists are able to make use of the digital media platform such as YouTube for disseminating the medical knowledge and benefits of organ donation (ibid). Meanwhile, new media has been argued to have played a crucial role in the promotion of sexual health (Guse et al. 2012). The ability to identify the health risks associated with unsafe sexual practices has accordingly been enhanced with the digitalisation of health communication (ibid). On the whole, all these three opportunities have pointed to the fact that the role of the new media in promoting public health goes beyond passive acquisition of health-related information; instead, it points to the possibility for a decentred, non-hegemonic way of disseminating and expanding the existing body of health knowledge. 


\section{Case Study: Social Media and the Promotion of Mental Health Literacy}

Whilst mental health has been a growing concern amongst many communities, public health knowledge about this issue has been unsystematic and poor in terms of quality (Jorm 2000; Reavley and Jorm 2013). As lamented by Jorm (2000), "members of the public cannot correctly recognise mental disorders and do not understand the meanings of psychiatric terms" (p. 396). This gives rise to the problem of miscommunication between the public and the medical professionals (ibid). Against this context, there has been a call for paying attention to mental health literacy. As argued by Reavley and Jorm (2013, p. 51), "although mental health literacy incorporates knowledge about mental disorders, it goes further in that it places emphasis on the knowledge being linked to the possibility of action to benefit one's own mental health or that of others." Mental health literacy should thus focus on "(1) knowing how to prevent mental disorders; (2) recognition of when a disorder is developing to facilitate early help-seeking; (3) knowledge of help-seeking options and available treatments; (4) knowing effective self-help strategies for milder problems; (5) first aid skills to support others who are developing a mental disorder or in a mental health crisis" (ibid, p. 51).

Meanwhile, how to operationalise the idea of mental health literacy is still a contested issue. One of the key opportunities since the $1990 \mathrm{~s}$ is the changing mode of communication which renders mental health no longer a 'taboo' to discuss. There have even been more initiatives taken by different communities to address the need to promote mental health literacy. Initiatives such as Mental Health Foundation and SANE Australia, for example, are well-established organisations in promoting mental health knowledge. SANE Australia, for example, has established a 'Lived Experience Forum' which provides 'a safe, anonymous place' for the public to share their concern (SANE Australia, n.d., a). Both Mental Health Foundation and SANE Australia have made full use of new media to disseminate knowledge about keeping mental healthiness. For example, the former organisation has designed free podcasts which introduce a variety of lifestyle tips for the public (Mental Health Organisation, n.d.). SANE Australia, on the other hand, has developed a guide to the media in order to avoid any further stigmatisation on those who are suffering from mental illness (SANE Australia, n.d., b).

Other initiatives take a more critical stance about the way in which mental health knowledge and information is constructed. Formed by a group of health activists, Hearing Voices Network (2015) has pointed to the 'unsoundness' and 'unreliability" of the contemporary approach of psychiatric diagnoses: "We believe that people with lived experience of diagnosis must be at the heart of any discussions about alternatives to the current system. People who use services are the true experts on how those services could be developed and delivered; they are the ones that know exactly what they need, what works well and what improvements need to be made" (Hearing Voices Network 2015). The Network organises an online platform on which those who are suffering from emotional disturbances can express 
themselves without emotional burdens. As asserted by Pilgrim and Rogers (2003), whilst initiatives of this kind may not be scientifically measurable, they do provide a possibility for 'emancipation' and for challenging the dominance of professional knowledge in understanding the medical condition of those who suffer from similar symptoms.

Open Access This chapter is distributed under the terms of the Creative Commons Attribution 4.0 International License (http://creativecommons.org/licenses/by/4.0/), which permits use, duplication, adaptation, distribution and reproduction in any medium or format, as long as you give appropriate credit to the original author(s) and the source, provide a link to the Creative Commons license and indicate if changes were made.

The images or other third party material in this chapter are included in the work's Creative Commons license, unless indicated otherwise in the credit line; if such material is not included in the work's Creative Commons license and the respective action is not permitted by statutory regulation, users will need to obtain permission from the license holder to duplicate, adapt or reproduce the material.

\section{References}

Adam, N.R., Shafiq, B. and Staffin, R., 2012. Spatial Computing and Social Media in the Context of Disaster Management, Intelligent Systems, IEEE, 27(6), 90-96.

Arksey, H., 1994. Expert and lay participation in the construction of medical knowledge, Sociology of Health and Illness, 13(1) 1-19.

Centers for Disease Control and Prevention (CDC), 2011, The Health Communicator's Social Media Toolkit, available at: http://www.cdc.gov/healthcommunication/ToolsTemplates/ SocialMediaToolkit_BM.pdf (accessed 12 August 2015).

Centers for Disease Control and Prevention (CDC), 2015. "Zombie preparedness," available at: http://www.cdc.gov/phpr/zombies.htm (accessed 16 August 2015).

Cromley, E. and McLafferty, S., 2012. GIS and Public Health, New York: The Guilford Press.

Davison, C., Smith, G., Frankel, S., 1991. Lay epidemiology and the prevention paradox: the implications of coronary candidacy for health education, Sociology of Health and Illness, 16(4), 448-468.

Dutta, M. and Zoller, H., 2008, Theoretical Foundations: interpretative, critical, and cultural approaches to health communication. In H. Zoller and M. J. Dutta, eds., Emerging Perspectives in Health Communication: Meaning, Culture, and Power, pp. 1-28. Oxon: Routledge.

Evans, J., Davies, B. and Rich, E., 2009. The body made flesh: Embodied learning and the corporeal device, British Journal of Sociology of Education, 30(4), 391-505.

Guse, K., Levine, D., Martins, S., Lira, A., Gaarde, J., Westmorland, W. and Gilliam, M., 2012. Interventions Using New Digital Media to Improve Adolescent Sexual Health: A Systematic Review, Journal of Adolescent Health, 51(6), 535-543.

Hagglund, K., Shigaki, C., and McCall, J. 2009. New Media: A Third Force in Health Care. In Jerry C. Parker, Esther Thorson (eds.), Health Communication in the New Media Landscape, pp. 417-36. New York : Springer.

Hamilton, H., and Chou, Wen-ying Sylvia eds. 2014. The Routledge Handbook of Language and Health Communication, Oxon: Routledge.

Harrison, T., Morgan S., King, A., et al., 2010. Promoting the Michigan Organ Donor Registry: Evaluating the Impact of a Multifaceted Intervention Utilizing Media Priming and Communication Design, Health Communication, 25(8), 700-708.

Hearing Voices Network 2015. Position Statement on DSM 5 \& Psychiatric Diagnosis, available at: http://www.hearing-voices.org/about-us/position-statement-on-dsm-5/ (accessed 17 August 2015). 
Hesse, B., Nelson, D., Kreps, G. et al., 2005. Trust and Sources of Health Information: The Impact of the Internet and Its Implications for Health Care Providers: Findings From the First Health Information National Trends Survey. Archives of Internal Medicine, 165(22), 2618-2624.

Jorm, A. 2000. Mental health literacy: public knowledge and beliefs about mental disorders, British Journal of Psychiatry, 177, 396-401.

Kickbusch, I. 2001. Health literacy: addressing the health and education divide, Health Promotion International, 16(3), 289-97.

Kickbusch, I. and Maag, D., 2008. Health Literacy. In K. Heggenhougen, S. Quah, eds., International encyclopaedia of public health, pp. 204-211. Boston : Elsevier.

Laverack, G. 2009. Public Health: Power, Empowerment and Professional Practice, Basingstoke, Hampshire: Palgrave Macmillan.

Meier A, Lyons E, Frydman G, Forlenza M and Rimer B, 2007. How Cancer Survivors Provide Support on Cancer-Related Internet Mailing Lists, Journal of Medical Internet Research, 9(2), e12.

Mental Health Organisation, (n.d.), Mental Health Organisation, available at: http://www. mentalhealth.org.uk/help-information/podcasts/ (accessed 12 August 2015).

Nutbeam, D., 2000. Health literacy as a public health goal: A challenge for contemporary health education and communication strategies into the 21 st century, Health Promotion International, 15(3), 259-267.

Nutbeam, D., 2008. The evolving concept of health literacy," Social Science and Medicine, 67(12) 2072-2078.

Parrott, R., Volkman, J. E., Lengerich, E., Ghetian, C. B., Chadwick, A. E. and Hopfer, S. 2010. Using Geographic Information Systems to Promote Community Involvement in Comprehensive Cancer Control, Health Communication, 25(3), 276-285.

Pilgrim, D., and Rogers, A., 2003, Mental Health and Inequality, Basingstoke, Hampshire: Palgrave Macmillan.

Prestin, A., and Chou, W-Y S. 2014, Web 2.0 and the changing health communication environment. In Heidi Hamilton, Wen-ying Sylvia Chou (eds.), The Routledge Handbook of Language and Health Communication, pp. 184-197. Oxon: Routledge,

Ratzan, S., Payne, J., and Schulte, S. 2004. Health communication. In N. Anderson (Ed.), Encyclopedia of Health and Behavior, pp. 398-402. Thousand Oaks, CA: SAGE Publications, Inc.

Reavley, N., and Jorm, A., 2013. Mental health literacy. In L. Knifton, and N. Quinn. Public Mental Health: Global Perspectives, pp. 50-58. Maidenhead, Open University Press.

SANE Australia, (n.d., a), "Lived Experience Forum," available at: http://saneforums.org/t5/LivedExperience-Forum/ct-p/lived-experience-forum (accessed 16 August 2015).

SANE Australia, (n.d.,b), "How media professionals can reduce the impact of prejudice and discrimination on people affected by mental illness," available at: https://www.sane.org/ images/media-centre/brochure_stigma_media.pdf (accessed 16 August 2015).

Sundar, S., Rice, R., Kim, H-S. and Sciamanna, C., 2011. Online Health Information. In T. Thompson, R. Parrott, and J. Nussbaum (eds.), Routledge Handbook of Health Communication ( $2^{\text {nd }}$ edition), pp. 181-202. Oxon: Routledge.

Thomas, R., 2006. Health Communication, New York: Springer.

Tian, Y. 2010. Organ Donation on Web 2.0: Content and Audience Analysis of Organ Donation Videos on YouTube. Health Communication, 25(3), 238-246. doi:10.1080/10410231003698911

World Health Organization, (n.d.), "Track 2: Health literacy and health behaviour," available at: http://www.who.int/healthpromotion/conferences/7gchp/track2/en/ (accessed 16 August 2015).

Zarcadoolas, C. and Pleasant, A., 2009. Health Literacy in the Digital World. In J. C. Parker, E. Thorson (eds.), Health Communication in the New Media Landscape, pp. 303-323. New York: Springer. 